\title{
Study on Rational Use of Tourism Occasion to Marketing Strategy
}

\author{
Sun Guoxue \\ College of Economics and Management, ChiFeng University, ChiFeng, Inner Mongolia, China (sgx500@163.com)
}

\begin{abstract}
Occasion to marketing as an effective marketing tool, More and more companies rely on. However, some tourism enterprises in the use of an occasion to marketing strategy, Due to various factors, There are significant limitations and problems, Even into errors. Occasion to marketing is difficult to improve the strategic level, Actual does not work effectively, Even self-destructive image. Reasonable use of occasion to marketing strategy, Must seriously study on occasion to marketing the connotation, Grasp its basic principles, definitude its inherent requirement, Flexible use of various types of occasion to marketing, To be able to achieve good market effect.
\end{abstract}

Key words--- Occasion to marketing, Basic Principles, Inherent requirements, Specific ways

\section{旅游借势营销策略合理运用研究}

\author{
孙国学 \\ 赤峰学院经济与管理学院, 赤峰, 内蒙古, 中国
}

摘 要 借势营销作为一种行之有效的营销利器, 为越来越多的企业所倚重。但是, 一些旅游企业在运用借势营销策略时, 直于 多方面因素的制约, 存在着很大的局限性和问题, 甚至走入误区。借势很难提高的战略层面, 实际效果不佳, 甚至自毁形象。正确合 理的运用借势营销策略, 必须认真研究借势营销的内涵, 把握其基本原则, 明确其内在要求, 对各类借势营销方式加以灵活的运用, 才能取得良好的市场效应。

关键词＼cjkstart借势营销，基本原则，内在要求，具体方式

\section{1. 引言}

营销活动中，利用企业自身资源以外的其他资源或渠 道来进行营销, 借助外在力量能够在营销过程中取得较高 的关注度和更好的营销效果。借势营销是将销售的目的隐 藏于营销活动之中, 将产品的推广融入到一个消费者喜闻 乐见的环境里, 使消费者在这个环境中了解产品并接受产 品的营销手段。具体表现为通过媒体争夺消费者眼球, 借 助消费者自身的传播力, 依靠轻松娱乐的方式等潜移默化 地引导市场消费。 ${ }^{[1]}$ 换言之, 便是通过顺势、借势、造势 等方式, 以求提高企业或产品的知名度、美誉度, 树立良 好的品牌形象, 并最终促成产品销售的营销策略。

这里所说的“借势”, 就是借助具有相当影响力的事件、 人物、产品、故事、传说、影视作品、社会潮流等, 策划
出对自己有利的新闻事件, 将自己带入话题中心, 由此引 起媒体和大众的关注, 让更多的人认识、关注自己, 提高 知名度。借他人之 “势” 为已所用, 这样就可以达到四两 拨千斤、事半功倍的神奇效果。

作为一种新型营销手段, 借势营销集新闻效应、广告 效应、公共关系、形象传播、客户关系于一体, 已经当之 无愧地成为了企业新产品推介、品牌展示、建立品牌识别 和品牌定位等营销活动的首选策略。据相关报道, 美国企 业在借势营销上的总花费年增长率高过 $15 \%$ 。企业 CEO、 CFO 等高层管理人员也越来越重视借势营销, 其中, 被调 查的高层决策人员中， $39 \%$ 的人很认同借势营销这种手段。

借势营销的优势在于: 一是受众者的信息接收程度较 高。借势营销避开了媒体多元化而形成的信息干扰, 受众 
对于其内容的信任程度、传播广度远远高于广告。调查表 明, 一个读者对新闻的信任程度是接受一则广告的六倍。 二是传播深度和层次高。一个事件如果成了热点, 会成为 人们津津乐道、互相沟通的话题, 事件被反复使用, 还可 以形成二次传播, 引发 “蝴蝶效应”。三是宣传成本低, 投 资回报率高。新闻事件传播面广、速度快、影响力大等特 点, 能有效节约企业建立品牌的成本。据统计分析, 企业 运用有创意的事件营销手段取得的投资回报率, 约为一般 传统广告的三倍[1]。

\section{2. 借势营销必须坚持的三项原则}

\section{1 事件的关联度}

在进行借势营销时, 要求与所借之势具有一定的关联 度。关联度越高, 说明借势的理由越充分, 而借势的理由 越充分, 则成功率越高。借势营销必须围绕一定的主题进 行, 关联度是进行借势营销活动成功的关键要素。当然, 这种关联度有时是直观的、直接的关系, 有的则是抽象的、 间接的关系。

借势营销能不能做好, 跟对 “势” 的准确判断有直接 关系。一个事件摆在眼前, 首先应该慎重判断到底能不能 借, 其根本是要看该事件对目前的推广目标有无帮助, 事 件与产品是否有关联性, 两者的内涵能否契合。借势营销 中存在不少盲目跟风的现象, 仅凭事件的高关注度就积极 地投身其中, 不管产品与 “势” 能否搭上关系, 就生搬硬 套地将二者硬拉到一起, 最终反而受到大家的置疑, 落个 “画虎不成反类犬” 的结局。

\section{2 公众的关注度}

借势营销主要是借助事件影响力营销, 影响力的大小 取决于人们对事件的关注度。很多企业进行借势营销, 投 入了不少资金, 却连个响声都听不见, 根本没有什么效果, 原因是关注度不够。不为公众所关注, 就不能吸引人们的 眼球, 就形成不了 “势”。

关注度主要是通过媒体报道提升的, 与以下几方面有 密切联系: 一是重要性。指事件内容的重要程度, 判断内 容重要与否的标准主要看其对社会产生影响的程度。一般 来说, 对越多的人产生越大的影响, 新闻价值越大。二是 接近性。越是心理上、利益上和地理上与受众接近和相关 的事实, 新闻价值越大。在策划事件营销时必须关注到你 的受众的接近性的特点。通常来说, 事件关联的点越集中, 就越能引起人们的注意。三是显著性。新闻中的人物、地
点和事件的知名程度越是著名, 新闻价值也越大。国家元 首、政府要人、知名人士、历史名城、古迹胜地往往都是 出新闻的地方。四是趣味性。人类本身就有天生的好奇心, 大多数受众对新奇、反常、变态、有人情味的东西比较感 兴趣。

\section{3 媒体的传播度}

借势营销对事件的曝光程度有很大的依赖。在大众传 播时代, 事件曝光程度取决于媒体的参与程度。事件营销 要达到预期效果, 需要媒体尤其是主流强势媒体的介入参 与, 这是吸引公众关注的前提。媒体参与的形式有两种: 一种是主动介入, 媒体如果认为此一事件有亮点、有卖点, 有新闻价值, 就会主动介入, 甚至持续跟踪报道。借势营 销的成功与否, 引爆点是至关重要的。引爆点就是借势营 销过程中能引起公众和媒体关注的事物。

一般来说, 突发事件易引起媒体主动介入, 旅游目的 地在利用突发事件进行营销宣传时, 要注意与媒体加强沟 通, 引导媒体有意识地进行旅游形象宣传。另一种是邀请 媒体参与, 最好在事件营销的策划阶段, 就邀请媒体合作, 共同参与策划, 以求取得最佳效果。相对于广播电视、报 纸杂志等传统媒体的单向传播特性来说, 互联网因其互动 特性, 成为当今与社会公众关系最为密切的传播媒体。“世 界最好工作” 事件就充分利用了互联网传播特性, 通过互 联网吸引了全球网民的关注与参与, 是该事件营销取得成 功的重要因素。

\section{3. 借势营销的内在要求}

\section{1 借势营销中的合作双赢}

传统的营销理念过于强调竞争，企业和相关企业之间 只是交易和竞争的关系。企业采取的竞争性战略往往是在 争夺同一块蛋糕, 这种你死我活的输赢之争, 不仅使企业 外部竞争环境恶化, 而且使企业错失许多良机。全球一体 化使得竞争格局发生了根本变化, 企业之间的竞争从有形 资产的竞争转向无形资产的竞争, 从价格、质量竞争转向 信息、人才竞争。顺应时代的发展, 企业越来越需要为竞 争而合作, 靠合作来竞争。合作竞争营销的理念, 使拥有 不同优势的企业在竞争的同时也注重彼此之间的合作, 通 过优势互补, 共同创造一块更大的蛋糕, 来实现 “双赢” 或 “多赢”。而在事件营销中进行不同行业和领域企业间的 合作则能扩大事件的影响, 提高企业的知名度, 扩大合作 双方彼此的影响力, 达到 “双赢” 或 “多赢” 的效果 [2]。 
例如中国湖南电视台 “超级女声” 是一个典型的多赢 的结局。超女活动不仅仅捧红了超女, 还给蒙牛带来了丰 厚的利润, 光是短信投票就让中国电信大挣一把, 高收视 率让湖南卫视巩固了其作为地方电视台龙头老大的地位。 这超女活动的事件营销给予我们太多的启示, 其中最为重 要的就是商业的本质是合作与赢利, 企业应与社会共赢共 生, 以开放的姿态与各方合作、互惠互利, 打造一个 “共 赢模式”, 这样的事件营销才能够取得足够的影响与成功。

\section{2 借势营销中的双向互动}

互动营销强调企业和消费者间交互式交流的双向推 动, 改变了传统营销中企业对消费者的单向推动。传统的 媒体广告、产品目录等只是企业单向地把产品信息输送给 消费者, 消费者完全被动地接收商品信息, 而企业也不能 及时获得消费者的反馈信息, 使得距离成为企业与消费者 之间交流的障碍, 企业难以及时、准确地了解顾客个性化 的需求。随着居民收入的提高、消费意识的成熟以及消费 理念的转化, 差异消费、个性消费成为时尚, 网络经济将 是一个个性化的客户关系的竞争模式。在事件营销中注重 与顾客互动的沟通与交流, 随时改进营销传播策略, 将成 为事件营销管理的关键。让消费者参与到产品制作与消费 的过程中去, 使得消费者获得比直接消费产品更大的满足 感与更为强烈的购买热情。互动营销在 “超级女声” 中表 现得淋漓尽致。海选使大众成为主宰选手命运的最重要因 素, 手机短信已经成为电视节目重头的利润来源。

\section{3 借势营销中的创意创新}

借势营销比较强调科学性, 更强调艺术性。效仿、跟 风取得的效应远不如原创的好。一般情况下, 人们遇到从 来没有遇到过的新鲜事物时, 会产生很大的注意力, 而且 更容易产生好感, 加深记忆。但人们再次看到类似事物的 时候却从潜意识里有种忽略和排斥感, 不会去关注和追捧。 所以借势营销要把握“创意创新” 原则, 讲求创意的独特性 和新颖性, 创造性地加以借势[3]。

\section{4. 借势营销的具体运作方式}

\section{1 借文化之势}

文化, 用一句简单的话来概括就是习性, 是特定人群 普遍自觉的观念和方式。比如在我国春节来临前, 不用动 员, 大家在用不同方式做着同一件事情, 朝着一个目标前 进一一回家。在中国人的概念中, 春节就要全家团圆, 这
种对春节普遍认同的观念和方式就是文化。这样一种统一 的行动, 不用号召, 不用法律规定, 为什么可以成为一种 自觉? 这背后是中国几千年的传统文化使然。所以说, 文 化是一种看不见的力量, 是一只看不见的巨手; 文化决定 着人们的价值观, 左右着人们的行动。文化的力量是思想 的力量, 是精神的力量, 是经典的力量, 是一个民族的重 量, 一个国家的分量, 一个社会的体温。

文化的力量同时也体现在旅游产品营销中。“上有天 堂, 下有苏杭”, 这句绝佳的广告词可谓是神来之笔, 不但 使苏州和杭州这两个 “人间天堂” 蜚声海内外, 还使两地 的旅游业大受其益。一首《无锡旅情》唱响一座城市, 一 幅《故乡的回忆》将周庄推向世界, 一首《枫桥夜泊》让 寒山寺的钟声传遍天下, 一部《非诚勿扰》使西溪湿地名 声大噪……这些旅游景区的功成名就显示了文化的力量。

\section{2 借影视之势}

影视也是一种文化, 更是一种特殊的文化形式。大量 事实表明, 旅游目的地形象的传播、推广和营销正是依靠 大众传媒来实现的, 是通过吸引公众的注意力从而产生 “眼 球经济” 效应。影视之所以能够宣传推广旅游目的地, 最 根本的当然是由于影视所固有的传播功能。电视、电影等 属于大众传媒, 影视文化会吸引公众注意力, 增强公众对 旅游目的地的好感度和美誉度, 使旅游者选择这些地方游 览并消费。影视作为一种新型的旅游营销方式, 已经成为 旅游营销和改善目的地形象的重要手段和媒介平台。

优秀的影视作品在客观上加强了外景地的吸引力, 是 卓有成效的宣传工具, 从而具有较高的旅游价值。影视作 品对旅游的促进作用主要表现在: 对拍摄地的旅游宣传、 带旺拍摄地的旅游人气、引导当地的旅游开发、宏扬当地 的历史文化、带来了综合效益。对很多人来说, 影视除了 提供物质和精神的享受外, 还能吸引他们到拍摄场地去旅 游。随着影视剧的播出, 可以使旅游目的地成为旅游热点。 调查显示, 超过 $20 \%$ 的人表示, 因为自己喜欢的影视剧在 当地拍摄, 会产生很强烈的旅行意愿, 另外还有 $23 \%$ 左右 的旅游爱好者也表示会有 “一定的冲动”。

决定影视能否带火旅游的有多种因素, 旅游之 “花” 能否如意绽放, 最核心的是片中出现的风景、人文会不会 激起人们由衷的向往。正是看到了影视在旅游宣传中存在 的巨大潜力, 以前 “无心插柳” 的意外效应, 逐渐变成了 “有心栽花” 的旅游营销方式。借助影视传播旅游也从被 动走向主动、从后期走向前期、从片面走向全面。

影视具有了传播旅游吸引物的功能, 但是在早期这种 
传播几乎是无意识的, 今天很多旅游目的地是主动地借助 于影视进行传播。在早期, 旅游目的地往往是借已经播映 的影视进行宣传, 这时影视已经拍摄好了, 进入后期播映 阶段。然而发展到今天, 很多旅游目的地事前就做好了利 用影视进行宣传营销的计划, 介入了影视产业早期的拍摄 选址乃至剧本写作阶段。北京市经过对国内外成功的旅游 影视营销案例进行深入研究和分析并结合北京的实际情 况, 最终决定采用与国内强势影视公司合作, 利用其优质 的影视资源通过各种营销手段进行事件营销来推广北京旅 游。在影视作品或活动中北京市旅游局都将以首席合作伙 伴的身份出现, 双方合作的内容也不仅仅局限在拍摄地点 的选择上, 而是充分发挥各自的优势资源以事件营销为载 体通过广告、媒体、公关等系统的手段推广北京旅游。

随着影视旅游的发展从被动走向主动, 从后期走向前 期, 影视和旅游两个产业的融合也从个别环节向全面融合 发展, 典型事物就是影视基地的诞生。在影视拍摄基地, 游客可以看到剧组拍摄的现场或遗留下来的场景、设施, 可以亲自体验影视作品中的食品、道具、服装及饰物, 过 把演戏瘾, 还可以体验飞檐走壁, 探寻特技效果制作的奥 秘。影视基地一方面可以用于影视拍摄制作, 另一方面则 是旅游活动的重要场所, 真正体现了影视产业和旅游产业 从产品、市场到企业、产业的融合[5]。

\section{3 借体育之势}

体育赛事是品牌最好的广告载体, 具有沟通对象数量 大、传播面广和针对性强等特点。人们对体育的热爱往往 是不计路途远近, 不惜花钱的。体育也正在成为旅游策划 的重要关注点, 借助体育赛事, 冠名、赞助广受人们关注 的重大体育活动和体育比赛来推广自己的品牌, 以吸引消 费者和媒体的眼球, 达到传播自己的目的。华东五市是传 统精品旅游线路, 但近年来遇冷。2010 年世博会在上海举 办, 华东五市旅游借助世博会契机再度火爆, 再创辉煌。

\section{4 借新闻之势}

企业利用社会上有价值、影响面广的新闻, 或者与相 关媒体合作, 不失时机地把自己的产品和新闻事件或消费 者身边的热点问题联系在一起, 发表大量介绍和宣传企业 产品或服务的软性文章进行报道, 以理性的手段传播自己, 从而吸引公众的视线, 来达到借力发力的传播效果。2009 年 12 月 27 日, 随着曹操高陵的发掘, 人们的视野聚焦到
了安阳西高穴村这个号称 “旅游价值不在秦始皇陵之下” 的曹操墓, 无疑是活的 “摇钱树”。河南安阳利用 “曹操墓” 大做文章, 要把曹操高陵建成三国文化考古、文物保护基 地和旅游景区, 并以此开展了一系列相关活动。

\section{5 借明星之势}

现代营销理论认为, 让消费者在众多相似的同类产品 中记忆其中一个产品是比较困难的, 但如果通过一个有特 点的公众人物来引导消费者记忆, 往往会起到良好的效果。 明星一向因为高知名度、高曝光率, 被看作是商品通向消 费者的最好介质。所以, 利用明星来宣传企业产品, 借此 提高产品知名度, 从而扩大产品的市场份额已被大大小小 的企业看作是销售促进的宝典。利用消费者爱屋及乌心理, 借助明星的知名度来加速品牌传播、加重产品的附加值, 借此培养消费者对该产品的感情、联想, 来赢得品牌忠诚 度。

现在是一个偶像的时代, 很多人有强烈的偶像崇拜的 心理。尤其是青少年, 在他们心中总是有那么一位或几位 名人偶像, 让其痴迷。他们不但喜欢明星偶像的一言一行、 一举一动、穿着打扮, 而且, 很多时候偶像还能成为他们 消费的意见领袖。于是, 明星们纷纷登上了广告的舞台, 向其 “粉丝” 推荐某个品牌或服务, 将 “迷” 对其的喜爱、 崇拜和尊敬转化为购买力和消费行为。

\section{参考文献(References)}

[1] Tan Xiaofang. Occasion to marketing: enterprises to enhance awareness of road and intraoperative, modern corporate culture, 2012.01, pp.68-70.

[2] Yang Yi. Tourism Marketing Integrated Marketing Communications in Theoretical study, Chinese trade, 2012.19, pp.188-189.

[3] Yang Xingyue. Our government tourism marketing innovation strategy, Development Research, 2007.11, pp.85-86.

[4] Li Hongling. Perspective drama occasion to marketing phenomenon, Market observation, 2011.01, pp.101-102.

[5] Wu Jinmei, Song Ziqian. Industry convergence Perspective Television Tourism Development, Tourism Tribune, 2011.06, pp.29-35. 